\title{
Multifunctional load-bearing hybrid hydrogel with combined drug release and photothermal conversion functions
}

\author{
Yulin Jiang ${ }^{1}$, Yutao Yang ${ }^{1}$, Xiaoyang Zheng ${ }^{2}$, Yong Yi ${ }^{2}$, Xianchun Chen ${ }^{1}$, Yubao Li ${ }^{1}$, Dan Sun ${ }^{3}$ and Li Zhang ${ }^{1}$
}

\begin{abstract}
The inability of damaged load-bearing cartilage to regenerate and self-repair remains a long-standing challenge in clinical settings. In the past, the use of PVA hydrogels as cartilage replacements has been explored; however, both pristine and annealed PVA are not ideal for load-bearing cartilage applications, and new materials with improved properties are highly desirable. In this work, we developed a novel hybrid hydrogel system consisting of glycerolmodified PVA hydrogel reinforced by a 3D printed PCL-graphene composite scaffold. The composition of the hydrogel within the hybrid material was optimized to achieve high water retention and enhanced stiffness. The hybrid hydrogel formed by reinforcement with a 3D printed PCL-graphene scaffold with optimized architecture demonstrated desirable mechanical properties (stiffness, toughness, and tribological properties) matching those of natural loadbearing cartilage. Our novel hydrogel system has also been designed to provide drug release and on-demand photothermal conversion functions and at the same time offers excellent biocompatibility with low cell adhesion. These promising properties may allow our unique hybrid hydrogel system to be used for potential applications, such as load-bearing cartilage repair/replacement, as well as targeting certain challenging clinical conditions, such as the treatment of severe arthritis.
\end{abstract}

\section{Introduction}

With the ever-increasing aging population worldwide and injuries from high-impact sports and accidents, the number of patients who suffer from cartilage damage is on the rise ${ }^{1}$. One report suggests that the global cartilage repair/regeneration market was $\sim$ USD 4.3 billion in 2017 and is expected to reach USD 6.5 billion by the end of $2024^{2}$. Unfortunately, cartilage is an avascular and nervefree tissue, and its healing/self-regeneration upon damage remains a significant clinical challenge ${ }^{3}$. To date, various hydrogel systems, such as injectable hydrogels ${ }^{4}$ and $3 \mathrm{D}$ printed cell-laden hydrogels ${ }^{5}$, have been deployed as bioscaffolds for articular cartilage tissue engineering ${ }^{6,7}$.

\footnotetext{
Correspondence: Dan Sun (d.sun@qub.ac.uk) or Li Zhang (nic1976@scu.edu.cn) ${ }^{1}$ Analytical and Testing Center, State Key Laboratory of Oral Diseases, \& School of Materials Science and Engineering, Sichuan University, Chengdu 610065, China ${ }^{2}$ School of Materials Science and Engineering, Southwest University of Science and Technology, Mianyang 621010, China
}

Full list of author information is available at the end of the article.
Despite recent developments, few such systems can offer desirable biomechanical properties matching those of natural tissues. Inspired by natural cartilage structures (i.e., proteoglycan gels reinforced by a fibrous collagen network $)^{8,9}$, researchers have also developed a number of hybrid material systems consisting of hydrogels with embedded woven scaffolds to offer improved mechanical properties. Despite their enhanced stiffness, the Young's modulus reported for such systems $\left(<1.5 \mathrm{MPa}^{10-12}\right)$ is insufficient for practical load-bearing applications, such as replacing damaged fibrocartilages in intervertebral discs, knee joints and temporomandibular joint $(\mathrm{TMJ})^{13}$. The use of other highly rigid reinforcement materials (such as alloys ${ }^{14}$ ) is not ideal, as they may induce a stress-shielding effect on the surrounding tissue ${ }^{15}$.

Poly(vinyl alcohol) (PVA)-based hydrogels have been widely used in the biomedical field. In recent years, such materials have attracted interest in artificial cartilage applications ${ }^{16,17}$. Unfortunately, as-prepared PVA hydrogels 
lack adequate stiffness, and as a result, they are normally annealed to achieve enhanced stiffness. However, this process significantly sacrifices water content, hence compromising the lubrication properties of the hydrogel ${ }^{18}$. Hydrophilic polymers such as polyacrylamide (PAM) $)^{19}$ and polyvinylpyrrolidone (PVP) ${ }^{20}$ have been introduced into PVA hydrogels to improve their water-holding properties; however, these modified hydrogels do not offer sufficient stiffness for load-bearing cartilage applications. On the other hand, incorporating graphene into poly( $\varepsilon$-caprolactone) (PCL) is known to enhance the mechanical properties of the polymer and improve its osteogenic capability $^{21,22}$. The exceptional photothermal conversion ability of graphene under NIR irradiation can be used for promising applications, such as photothermal therapy in cancer treatments ${ }^{23}$. However, this unique property has rarely been explored in the context of tissue engineering/ cartilage repair.

Considering the growing demands for biomaterials that can play multiple biological roles in the development of modern therapies, we designed and fabricated a novel hybrid hydrogel system consisting of glycerol-modified PVA hydrogel reinforced by a 3D printed PCL-graphene scaffold, namely, PG-Pg. The PCL-graphene (PG) scaffold is completely encapsulated within the PVA-glycerol (Pg) hydrogel, where the Pg hydrogel provides lubricity and drug loading/release function, while the PG scaffold offers mechanical enhancement and photothermal effects. With its optimized design, our hybrid hydrogel features excellent biocompatibility, minimal protein/cell adhesion, exceptionally high water content, and desirable mechanical properties, making it suitable for load-bearing cartilage applications. The hybrid hydrogel also demonstrates effective photothermal conversion under NIR laser irradiation and drug release. This study provides a unique and sensitive route to control the hybrid hydrogel structure/properties/function necessary to meet the demanding requirements of load-bearing cartilage replacement. In addition, this is the first time a scaffold with multimodal therapeutic functions has been developed to target challenging joint diseases such as severe arthritis.

\section{Materials and methods \\ Materials}

PCL $(\mathrm{Mw}=80000)$ powder was purchased from Shenzhen Esun Industrial Co., Ltd, China. Dichloromethane $\left(\mathrm{CH}_{2} \mathrm{Cl}_{2}\right.$, A.R. grade) was purchased from Chengdu Changlian Chemical Reagent Co., Ltd, China. Poly(vinyl alcohol) (PVA, polymerization degree $=1799 \pm 50$, hydrolysis $>$ $90.5 \%)$, glycerol $\left(\mathrm{C}_{3} \mathrm{H}_{8} \mathrm{O}_{3}\right.$, A.R. grade) and anhydrous ethanol (A.R. grade) were purchased from Chengdu Kelong Co., China. Graphene was obtained from The Sixth Element (Changzhou) Materials Technology Co., Ltd, China. Sodium fluorescein (FL) was obtained from Xiya Reagent Co., Ltd., China. Phosphate buffer saline (PBS) tablets were purchased from Beijing Solarbio Science \& Technology Co., Ltd, China. All reagents were analytical grade.

\section{Preparation of hybrid hydrogel}

The preparation of the hybrid hydrogel is presented in Scheme 1. PCL powder $(5 \mathrm{~g})$ and graphene $(0 \mathrm{~g}$ and $0.05 \mathrm{~g})$

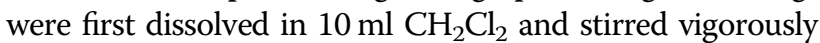
for $30 \mathrm{~min}$. The uniform PCL and PCL-G solutions were then transferred to a bioprinter (3D BioprinterV2.0, China)

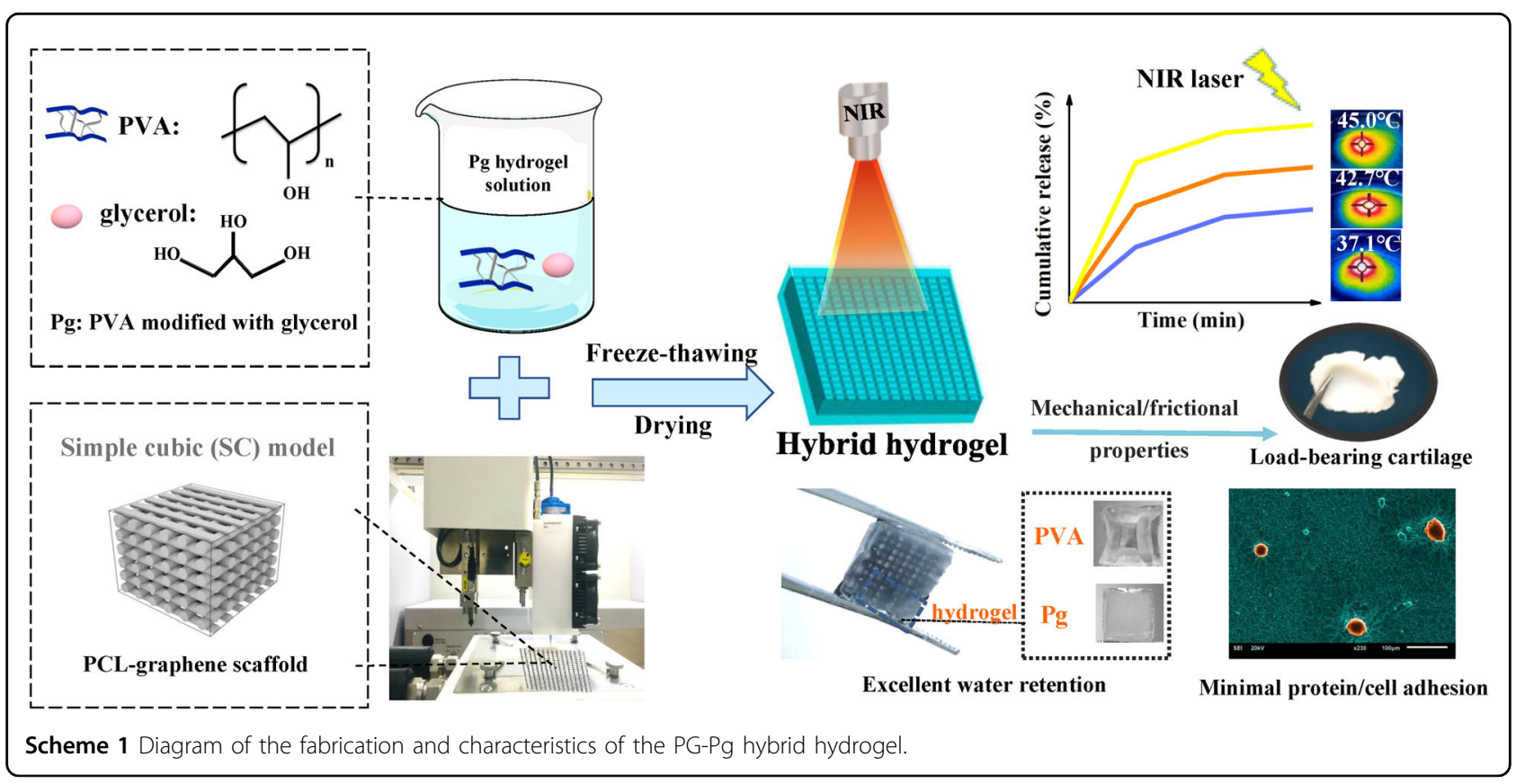


reservoir for 3D printing. The printing speed was $2 \mathrm{~mm} / \mathrm{s}$, the strut diameter was $0.21 \mathrm{~mm}$, the printing resolution was $0.1 \mathrm{~mm}$ and the strut spacing was $0.6,0.8$ and $1.0 \mathrm{~mm}$. The printed pure PCL scaffolds were named PCL-0.6, PCL-0.8, and PCL-1.0, and the PCL-graphene scaffolds were named PCL-G-0.6, PCL-G-0.8, and PCL-G-1.0. The printed PCL and PCL-G 3D structures were dried at room temperature in a vacuum oven to allow complete evaporation of the residual $\mathrm{CH}_{2} \mathrm{Cl}_{2}$. A $15 \mathrm{wt} \%$ PVA solution was obtained by dissolving PVA powder in deionized water and stirring at $90^{\circ} \mathrm{C}$ for $6 \mathrm{~h}$. Appropriate amounts of glycerol were then added to the PVA solution to obtain PVA/glycerol mixtures with varying glycerol concentrations $(0,10 \mathrm{wt} \%, 20 \mathrm{wt} \%$ and $30 \mathrm{wt} \%)$. The resulting hydrogel mixtures were named Pg-0, Pg-10, Pg-20 and Pg-30, respectively. Next, the printed PCL and PCL-G 3D structures were infused with the PVA/glycerol mixture solutions under negative pressure to ensure thorough infiltration of the hydrogel into the interior of the scaffold pores. Afterwards, the hybrid hydrogels were subjected to three freezing-thawing cycles $\left(12 \mathrm{~h}\right.$ at $-20^{\circ} \mathrm{C}$ and $2 \mathrm{~h}$ at room temperature) to achieve crosslinking of the hydrogel network. The resulting hybrid hydrogels were dried at $50^{\circ} \mathrm{C}$ for $5 \mathrm{~h}$ and named PG-Pg. Pg hydrogels (i.e., without 3D printed reinforcement) with varying glycerol contents were also dried at $50^{\circ} \mathrm{C}$ for $5 \mathrm{~h}$ (Pg- $5 \mathrm{~h}$ ) for comparison study and were named Pg-0-5h, Pg-10-5 h, Pg-20$5 \mathrm{~h}$ and Pg-30-5h.

\section{Characterization}

Scanning electron microscopy (SEM, JEOL, JEM6500LV, Japan) and a stereomicroscope (Nikon SMZ800, Japan) were used to observe the morphology of all samples. The crystallinity of the Pg hydrogel was examined by X-ray diffraction (XRD, DX-2500, China). Fourier transform infrared spectroscopy (FTIR, Nicolet-6700, USA) was performed in the range of $4000-500 \mathrm{~cm}^{-1}$. Differential scanning calorimetry (DSC) and thermogravimetric analysis (TGA) were carried out using a TGA/DSC/1600 (Star System, Mettler Toledo). Quantitative elemental analysis was carried out using X-ray photoelectron spectroscopy (XPS, XSAM800 UK).

\section{Porosity measurement}

The porosity $\left(P_{\mathrm{s}}\right)$ of the PCL and PCL-G scaffolds was determined by the liquid substitution method. The samples $\left(10 \times 10 \times 6 \mathrm{~mm}^{3}\right)$ were immersed in anhydrous ethanol and repeatedly pumped until anhydrous ethanol was completely filled in the scaffolds. The porosity was calculated following Eq. (1):

$$
\operatorname{Porosity}(\%)=\left(\mathrm{W}_{1}-\mathrm{W}_{0}\right) /(\mathrm{V} . \rho) \times 100 \%
$$

where $\mathrm{W}_{0}$ and $\mathrm{W}_{1}$ are the weight of the scaffolds before and after pumping, respectively, $\mathrm{V}$ is the volume of the scaffolds, and $\rho$ is the density of anhydrous ethanol. Five samples were tested, and the mean value was taken for analysis.

\section{Water content}

The water content $\left(\mathrm{W}_{\mathrm{C}}\right)$ of the hydrogels was measured by the gravimetric method. The samples $(10 \times 10 \times$ $6 \mathrm{~mm}^{3}$ ) were dried at $50^{\circ} \mathrm{C}$ until no further weight loss occurred. $W_{C}$ was calculated following Eq. (2):

$$
\mathrm{W}_{\mathrm{C}} \%=\left(\mathrm{W}_{0}-\mathrm{W}_{1}\right) / \mathrm{W}_{0} \times 100 \%
$$

where $\mathrm{W}_{0}$ and $\mathrm{W}_{1}$ are the weight of the hydrogel before and after drying, respectively. Surface moisture was removed from the wet hydrogels by filter paper prior to measurement. Five samples were tested, and the mean value was taken for analysis.

\section{Swelling ratio}

The dried samples were immersed in distilled water, and the weight of the swollen hydrogels was measured at different time intervals (i.e., $2 \mathrm{~h}, 1$ day, 3 days, 5 days, and 7 days) until no further weight gain was observed. The swelling ratio $\left(\mathrm{W}_{\mathrm{S}}\right)$ was calculated according to Eq. (3):

$$
\mathrm{W}_{\mathrm{s}} \%=\left(\mathrm{W}_{2}-\mathrm{W}_{1}\right) / \mathrm{W}_{2} \times 100 \%
$$

where $\mathrm{W}_{2}$ and $\mathrm{W}_{1}$ are the weight of the hydrogel in the dehydrated and swollen states, respectively. Surface moisture was removed from the wet hydrogels by filter paper prior to measurement. Five samples were tested, and the mean value was taken for analysis.

\section{Finite element simulation}

As the PCL-graphene (PCL-G) scaffolds were 3D printed by the direct writing technique, two typical lattice models, namely, face-centered tetragonal (FCT) and simple cubic (SC) models, were adopted. Before fabricating the hybrid hydrogel systems, a finite volume method (FVM) simulation was carried out to investigate the effects of Pg-20-5h hydrogel infiltration on the mechanical performance of the 3D printed scaffolds and probe the load-bearing behavior of the hybrid systems under uniaxial compression conditions. Quasi-static uniaxial compression simulations were performed in the $z$-axial direction via a commercial software package, GeoDect ${ }^{24}$.

\section{Mechanical tests}

Uniaxial compression and tensile testing of all test samples were carried out following the standards $\mathrm{GB} / \mathrm{T}$ 1041-2008 and GB/T1040.2-2006 using a universal testing machine (INSTRON, American). A strain rate of $1 \mathrm{~mm} /$ min was applied until a predetermined compression ratio 
of $40 \%$ was reached, and the samples were stretched at a speed of $50 \mathrm{~mm} / \mathrm{min}$ under ambient conditions. Five samples from each group were tested, and the mean value was obtained for further analysis. The samples from different groups were also tested on a universal testing machine (AGS-X, Japan) for their creep behavior. The load was set to $5 \mathrm{~N}(0.05 \mathrm{MPa})$ at a speed of $10 \mathrm{~N} / \mathrm{s}$, and the dwelling period was $3600 \mathrm{~s}$. The load was then reduced to $1 \mathrm{~N}(0.01 \mathrm{MPa})$ at a speed of $10 \mathrm{~N} / \mathrm{s}$ and held for $3600 \mathrm{~s}$. The tensile loading/unloading behaviors of the materials were investigated by subjecting the samples to cyclic loading (stretching to $200 \%$ strain and unloading to $0 \%$ strain at a tensile strain speed of $50 \mathrm{~mm} / \mathrm{min}$ ) for five cycles.

\section{NIR laser-induced photothermal performance}

The samples were irradiated by an $808 \mathrm{~nm}$ near-infrared (NIR) laser under various power densities $\left(0-0.8 \mathrm{~W} / \mathrm{cm}^{2}\right)$ for different durations $(0-5 \mathrm{~min})$. The temperature of the irradiated samples was recorded using an infrared thermal camera (FLIR E6).

\section{Drug loading and release}

FL (0.1 wt\%) was loaded into the PVA/glycerol mixture solution during the hydrogel synthesis process. The FL solutions were diluted to gradient concentrations to establish a standard drug release curve using the absorbance data from UV-vis spectroscopy. The drug-laden hydrogels were immersed in $10 \mathrm{~mL}$ of PBS ( $\mathrm{pH}=7.4)$, and the cumulative release was measured with and without laser irradiation using UV-vis. The drug encapsulation efficiency was calculated by the following Eq. (4):

$$
\text { Encapsulation efficiency }(\%)=\left(C_{2}-C_{1}\right) / C_{2} \times 100 \%
$$

where $C_{1}$ and $C_{2}$ are the amount of unbound FL and total added FL, respectively.

\section{Tribological tests}

The reciprocal sliding test was performed on $30 \times 7 \times$ $6 \mathrm{~mm}^{3}$ hydrogel samples using a ball-on-plate highfrequency tribometer (MDW-02, China). Both PG-Pg and goat TMJ discs were slid against a static titanium alloy ball (diameter of $6.35 \mathrm{~mm}$ ) at $20 \mathrm{~mm}$ per second. The load applied was $5 \mathrm{~N}$, and the frictional coefficient of each specimen was measured for $30 \mathrm{~min}$. Each test was repeated three times, and the average friction coefficients were recorded for further analysis. Finally, the worn surfaces were examined by scanning electron microscopy (SEM, JEOL, JEM-6500LV, Japan) for morphological characterization.

\section{In vitro cell viability}

All animal experiments were conducted in accordance with international guidance on animal welfare and the standards of the Animal Research Committee of the State Key Laboratory of Oral Diseases and West China School of Stomatology, Sichuan University (approval number: WCCSIRB-D-2014-407). Intact TMJ discs were dissected from adult goats (each goat weighed $\sim 30 \mathrm{~kg}$ ). The preliminary biological properties were evaluated by cell coculture experiments. Briefly, chondrocytes were separated from fresh knee cartilage of newborn rabbits. The tissue was cut into slices and digested in $0.25 \%$ trypsin (Gibco, USA) at $37^{\circ} \mathrm{C}$ for $30 \mathrm{~min}$, followed by $0.3 \%$ type II collagenase (Gibco, USA) for $3 \mathrm{~h}$. After centrifugation, the cells were cultured in Dulbecco's modified Eagle's medium (DMEM; $4.5 \mathrm{~g} / \mathrm{L}$ glucose; Invitrogen, CA, USA) supplemented with $20 \%$ fetal bovine serum (FBS, Gibco, Canada) and $1 \%$ streptomycin and penicillin (HyClone, USA). The cells were incubated at $37^{\circ} \mathrm{C}$ in an atmosphere containing $5 \% \mathrm{CO}_{2}$. The cells of the 3rd passage were collected for detection by CCK8 and SEM.

The dried hydrogel samples (Pg-0-5 h to Pg-30-5 h and PG-Pg) were shaped into discs whose size matched that of the wells of a 48-well plate. Cells were cultured in 48-well plates at $10^{5} / \mathrm{ml}$ at $37^{\circ} \mathrm{C}$ and $5 \% \mathrm{CO}_{2}$ on the surface of the samples. After $48 \mathrm{~h}$ incubation, the cells were fixed with 2.5\% glutaraldehyde, washed with PBS solution and dehydrated using ethanol at graded concentrations (30, 50, 75, 90, and 100\%). The morphology and adhesion of chondrocytes were observed by SEM. Cell viability was evaluated by cell counting kit-8 (CCK- 8 ) assays. The sterilized disc-shaped samples were placed in a 48-well plate containing $300 \mu \mathrm{l}$ chondrocytes at $5 \times 10^{4} / \mathrm{ml}$. After culturing for 2 and 5 days the cell-laden hydrogels were washed with PBS and immersed in $300 \mu \mathrm{l}$ fresh medium topped with $30 \mu \mathrm{l}$ CCK8 solution in each well. The optical density (OD) was measured at an excitation wavelength of $450 \mathrm{~nm}$ by an Anthos 2010 spectrophotometer (Biochrom, Cambridge, UK). The cytotoxicity of PG-Pg was tested by measuring chondrocyte proliferation in cell culture medium mixed with leaching solution.

\section{Results and discussion \\ Characterization of $\mathrm{Pg}$ hydrogels}

Pg hydrogels were synthesized by adding various concentrations of glycerol $(0,10 \%, 20 \%$, and $30 \%)$ into PVA solution, and the resulting samples were named Pg-0, Pg10, Pg-20, and Pg-30, respectively. FTIR spectra of the Pg samples are shown in Fig. 1a, (a). The wide absorption peak at $3277-3253 \mathrm{~cm}^{-1}$ can be attributed to $\mathrm{O}-\mathrm{H}$ stretching vibrations in neat PVA and glycerol. As the glycerol content increased, the absorption peak redshifted due to the formation of new hydrogen bonds between glycerol and PVA $^{25}$ (as shown in Fig. 1a, (c). Such 


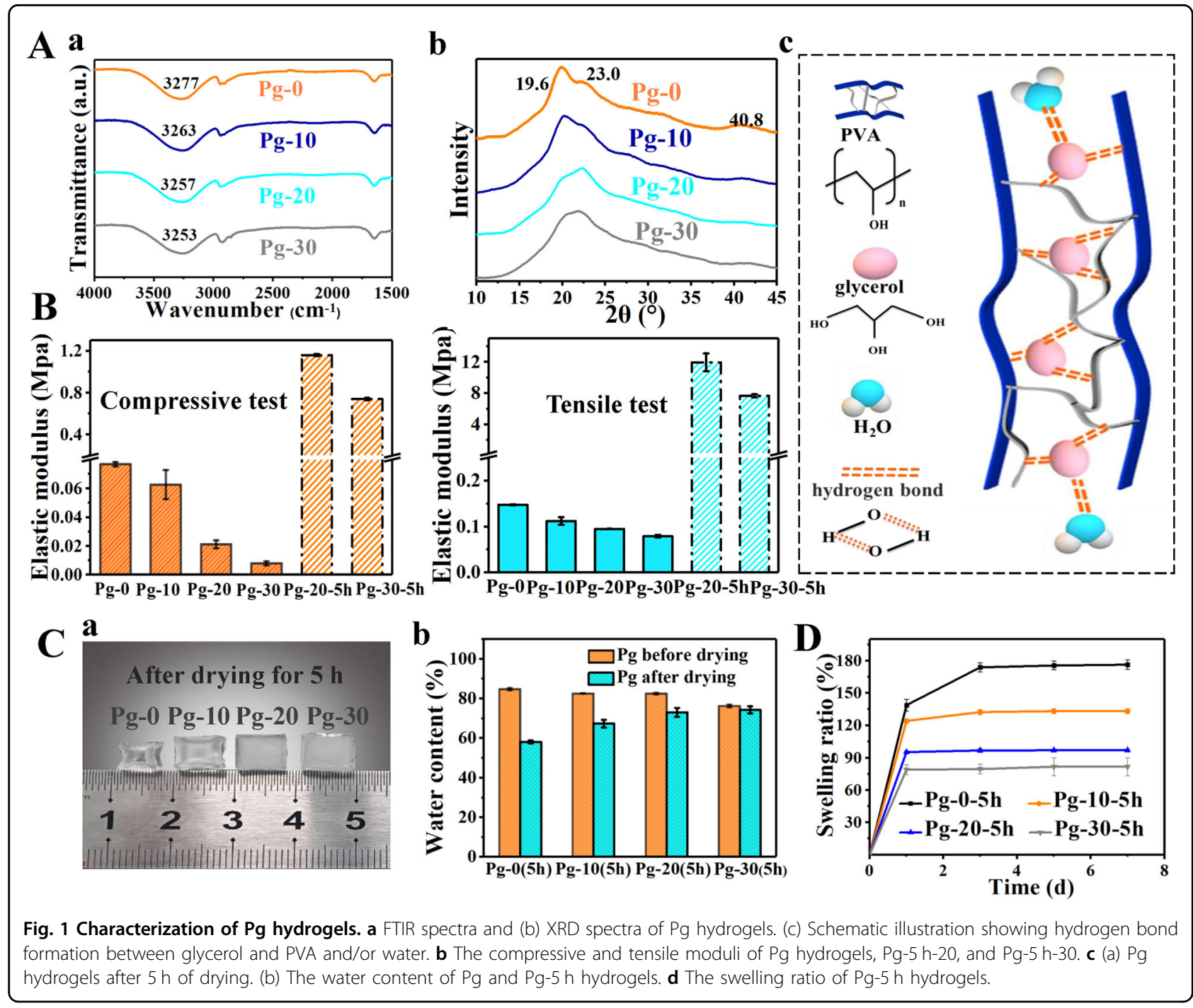

hydrogen bond formation reduces the force constants of $-\mathrm{OH}$ groups ${ }^{26}$ and reduces the microcrystalline region within the PVA, leading to increased distance and mobility of the molecular chains ${ }^{27}$. From the XRD data in Fig. 1a (b), it can be seen that the major peaks at $2 \theta=$ $19.6^{\circ}$ correspond to the typical crystallization of the irregular PVA $(10 \overline{1})$ plane. The shoulder peak at $2 \theta=22.9^{\circ}$ and the weaker diffraction peak at $2 \theta=40.8^{\circ}$ correspond to the PVA (200) and (102) planes, respectively ${ }^{28,29}$. The decreased PVA crystallinity can be evidenced by the broadening of the characteristic XRD peak at $2 \theta=19.6^{\circ}$. The mechanical testing results in Fig. $1 \mathrm{~b}$ suggest that increasing glycerol content (and hence decreasing crystallinity) gradually lowered the compressive and tensile moduli of Pg, the values of which were merely $0.021 \pm$ 0.002 and $0.095 \pm 0.001 \mathrm{MPa}$, respectively, for Pg-20, as shown by the solid bars in Fig. 1b.

To optimize the stiffness and tensile moduli of the Pg hydrogels, samples were dried at $50^{\circ} \mathrm{C}$ for $5 \mathrm{~h}$ for further studies. Figure 1c, (a) shows that Pg-0 underwent significant shrinkage after drying and Pg-10 showed mild shrinkage. In contrast, Pg-20 and Pg-30 maintained more intact shapes. This result is consistent with the data shown in Fig. 1c, (b), where an increasing glycerol content allowed better water retention during the drying process. This behavior can be attributed to the $-\mathrm{OH}$ functional groups of glycerol interacting with the $-\mathrm{OH}$ groups of water molecules through hydrogen bonds, hence effectively binding water within the hydrogel network ${ }^{30}$, as illustrated by Fig. 1a, (c). Figure 1d shows that the swelling ratio of all samples stabilized from 3 days, and samples with increasing glycerol content exhibited a lower swelling ratio, which is desirable for load-bearing cartilage applications. In addition, the drying process significantly improved the compressive and tensile moduli of the materials (Fig. 1b, dashed bar), which peaked for Pg-20$5 \mathrm{~h} \quad(1.16 \pm 0.01 \mathrm{MPa} ; 11.96 \pm 1.12 \mathrm{MPa})$. The thermal program (holding at $50^{\circ} \mathrm{C}$ for $5 \mathrm{~h}$ and then slowly cooling 
to room temperature) enhanced the mobility of PVA chains and generated a much higher density of PVA crystallites $^{18,19}$. From Fig. S1B, both Pg-20 and Pg-20-5 h showed endothermic peaks at $\sim 100^{\circ} \mathrm{C}$ as a result of water evaporation. The melting peaks of the Pg hydrogels appear at $200-30{ }^{\circ} \mathrm{C}^{31}$, and the greater endothermicity of Pg-20-5 h can be associated with its higher crystallinity. At melting temperature, the weight reduction of the samples (see Fig. S1A) is due to the evaporation of glycerol. From Fig. S1A, the weight loss between room temperature and $100^{\circ} \mathrm{C}$ is due to water evaporation. The weight loss between $120^{\circ} \mathrm{C}$ and $250^{\circ} \mathrm{C}$ is due to glycerol evaporation, and the weight loss occurring from $270-400^{\circ} \mathrm{C}$ is caused by the decomposition of glycerol. The DSC data suggest that the melting temperature of Pg$20-5 \mathrm{~h}$ is $\sim 260^{\circ} \mathrm{C}$, indicating its good thermal stability.

Given the superior mechanical properties of Pg-20-5 h among all the Pg hydrogel samples prepared, it was selected for preparation of a demonstration PG-Pg hybrid hydrogel for further investigation.

\section{Characterization of the PG-Pg hybrid hydrogel}

As shown in Fig. 2a, in the original FCT structure, each 3D printed scaffold layer was shifted by half the lattice spacing in the $\mathrm{XY}$ plane, resulting in a zigzag strain concentration under compression. Nevertheless, in the presence of the Pg-20-5h hydrogel, the strain concentration area shifted towards the regions filled by the low-stiffness hydrogel. In the original SC structure, on the other hand, the transverse and longitudinal struts intersected repetitively, leading to columnar strain concentration following a rectangular array pattern. When the Pg hydrogel was introduced, the strain concentration became more uniform, with only a slightly greater stress concentration at strut interconnections. Overall, hydrogel infiltration contributes to strain concentration transfer, thus resulting in a hybrid system with generally enhanced stiffness and strength. It is noteworthy that the effective Young's modulus of the SC configuration was approximately $15 \%$ higher than that of the FCT configuration; thus, we selected the more rigid SC structure to produce the 3D printed PCL-G scaffolds.

SC PCL and PCL-G scaffolds with different strut spacings $(0.6,0.8$, and $1.0 \mathrm{~mm}$ ) were fabricated (named PCL0.6, PCL-0.8 and PCL-1.0; and PCL-G-0.6, PCL-G-0.8 and PCL-G-1.0, respectively). The corresponding porosities were $58 \pm 4 \%, 63 \pm 3 \%$, and $80 \pm 2 \%$, respectively (see Fig. S2A). Figure S2Ba shows the typical compressive stressstrain curves of 3D printed PCL and PCL-G scaffolds with varying strut spacings. Figure S2Bb (elastic modulus) confirms that the addition of graphene enhanced the stiffness of the PCL scaffolds. In addition, the scaffold compressive moduli decreased with increasing strut spacing (i.e., increasing porosity from $58 \%$ to $80 \%$ ). Larger

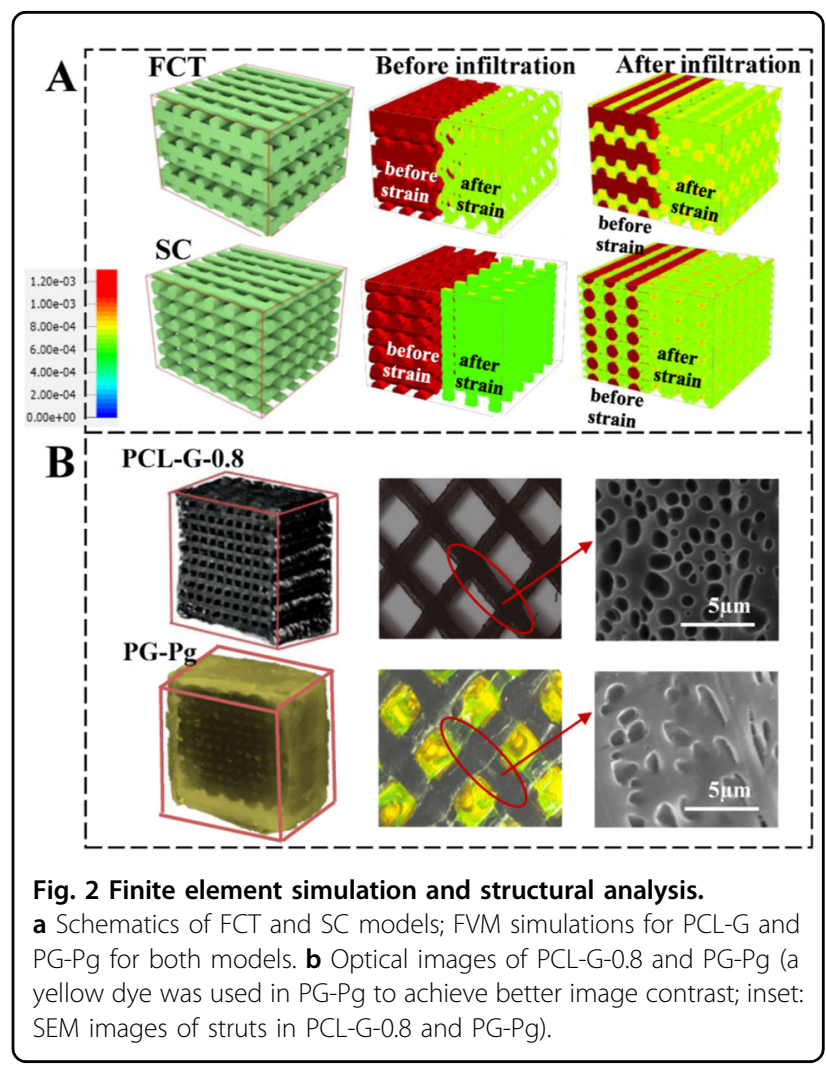

pores allowed easier infiltration and hence the formation of a more intact hybrid hydrogel structure. In contrast, oversized pores $(1.0 \mathrm{~mm})$ caused a sharp loss in stiffness (see PCL-G-1.0 in Fig. S2Bb). The PCL-G-0.8 scaffold was selected as reinforcement for the PG-Pg hybrid hydrogel owing to its modest stiffness.

The Pg-20-5 h hydrogel was infiltrated into the PCL-G0.8 scaffold to obtain the demonstration hybrid hydrogel (named PG-Pg). The optical images in Fig. 2b confirm that the 3D printed scaffold was well encapsulated in the hydrogel. Irregular pores of $1-3 \mu \mathrm{m}$ were present on the scaffold strut surface (see SEM inset), which were formed due to solvent evaporation during scaffold preparation. This strut surface morphology may facilitate mechanical interlocking between the PCL-G-0.8 scaffold and the infiltrated Pg-20-5 h hydrogels.

Mechanical testing of the hybrid hydrogel (PG-Pg) was carried out, and the results were compared with those for the PCL-G-0.8 scaffold and Pg-20-5h (see Fig. 3a). The compressive modulus of PG-Pg was lower than that of the pure PCL-G-0.8 scaffold (due to the buffering effect of the surface hydrogel) but was significantly greater than that of Pg-20-5 h. In contrast, the tensile strength and modulus of PG-Pg $(4.41 \pm 0.12 \mathrm{MPa}$ and $9.53 \pm 0.21 \mathrm{MPa}$, respectively) were considerably higher than those of PCL-G-0.8 (1.12 \pm $0.09 \mathrm{MPa}$ and $1.78 \pm 0.11 \mathrm{MPa}$ ). From Fig. 3b, it is worth noting that PG-Pg had a similar stiffness $(4.2 \mathrm{MPa})$ to that 

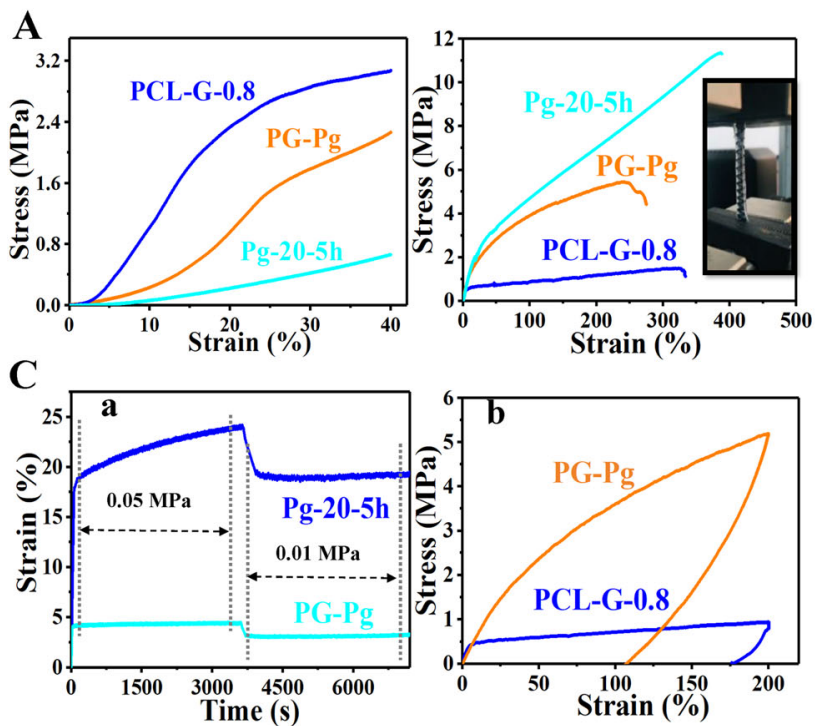

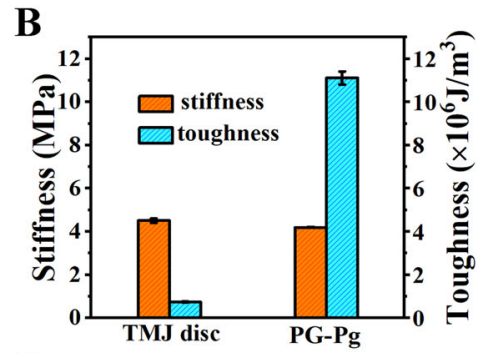

D

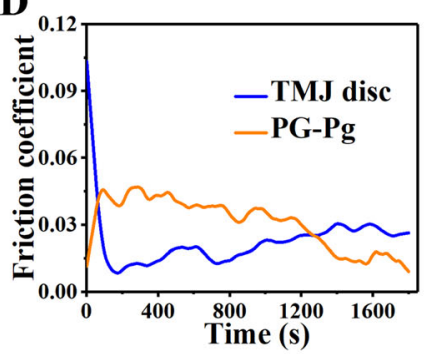

Fig. 3 Mechanical analysis. a The compressive and tensile stress-strain curves of Pg-20-5 h, PCL-G-0.8 and PG-Pg (inset: PG-Pg under tensile testing). b The stiffness and toughness of goat TMJ disc and PG-Pg. c (a) Strain vs. time curves of Pg-20-5 h and PG-Pg. (b) Loading/unloading stress-strain curves of PCL-G-0.8 and PG-Pg. $\mathbf{d}$ The friction coefficient curves of goat TMJ disc and PG-Pg.

of goat TMJ disc and at the same time offers a much better toughness $\left(1.11 \times 10^{7} \mathrm{~J} / \mathrm{m}^{3}\right)$. The sample creep resistance and recovery behavior can be found in Fig. 3c. Under constant loading conditions (0.05 MPa for $3600 \mathrm{~s})$, Pg-20$5 \mathrm{~h}$ first showed a significant increase in strain $(\sim 17 \%)$, which further increased steadily with time. The strain of PG-Pg, on the other hand, was much lower (5\%) under the same loading condition and was less time-dependent, indicating its much better creep resistance. In addition, both samples showed similar recovery dynamics. The energy dissipation behavior of the hybrid hydrogel and PCL-G0.8 scaffold during cyclic loading is shown in Fig. 3c (b). Due to the inevitable plastic deformation and internal friction, the hysteresis loop of the PCL-G-0.8 scaffold is not well enclosed. In contrast, PG-Pg exhibited much better recovery. Furthermore, the tribological properties of PG-Pg and goat TMJ discs are compared in Fig. 3d. The value of the frictional coefficient for PG-Pg gradually decreased and approached that of the goat TMJ disc at $\sim 1200 \mathrm{~s}$. The friction further decreased as the test progressed, which may be due to the lubrication effect of glycerol leached out from the hydrogel during testing. The PG-Pg worn surface features uniform wear tracks with no obvious cracks or fractures (see Fig. S3). Furthermore, the water content of the PG-Pg hybrid hydrogel reached $72 \pm 1 \%$, which is comparable to that of natural articular cartilage $\left(\sim 60 \%-80 \%^{32}\right)$.

\section{Photothermal conversion of PG-Pg}

Structural damage to load-bearing cartilage may lead to severe inflammatory joint conditions such as rheumatoid arthritis (RA). The literature suggests that combining localized photothermal therapy and drug release can inhibit inflammation well and hence maximize therapeutic efficacy and minimize dosage-related side effects in the treatment of $\mathrm{RA}^{33}$. We designed and fabricated a hybrid hydrogel with NIR light sensitivity coupled with a drug release function. The NIR light sensitivity can be attributed to the two-dimensional (2D) graphene sheet with a structure of sp2-hybridized carbon atoms packed into a honeycomb lattice. The unique electronic structure of graphene enables its strong interaction with photons at frequencies ranging from infrared to terahertz ${ }^{34}$.

Figure 4a, (a) shows the photothermal effect of the PG-Pg hybrid hydrogels under laser irradiation with different power densities. As shown in Fig. 4a, (b), for all laser densities, the temperature of PG-Pg rose sharply during the initial period. The sample temperature increased with irradiation laser power density $\left(0.07-0.31 \mathrm{~W} / \mathrm{cm}^{2}\right)$ and tended to stabilize after $\sim 50 \mathrm{~s}$. In particular, the temperature of the samples irradiated with a laser power density of $0.31 \mathrm{~W} / \mathrm{cm}^{2}$ reached $\sim 43{ }^{\circ} \mathrm{C}$ within $50 \mathrm{~s}$. It is worth noting that for PCL-G-0.8 (without hydrogel), high power densities $\left(0.19\right.$ and $\left.0.31 \mathrm{~W} / \mathrm{cm}^{2}\right)$ can lead to a temperature exceeding the PCL melting temperature and damage the 3D printed scaffold (see Fig. S4B). Figure 4a, (c) shows that under repeated laser irradiation, the photothermal performance of PG-Pg did not deteriorate, demonstrating its excellent thermal stability in repeated applications. The photothermal conversion of PG-Pg hybrid hydrogels was also investigated in PBS. Figure $4 \mathrm{~b}$ shows that for samples immersed in PBS to achieve a therapeutic temperature window, greater laser power densities were required. More specifically, the power 

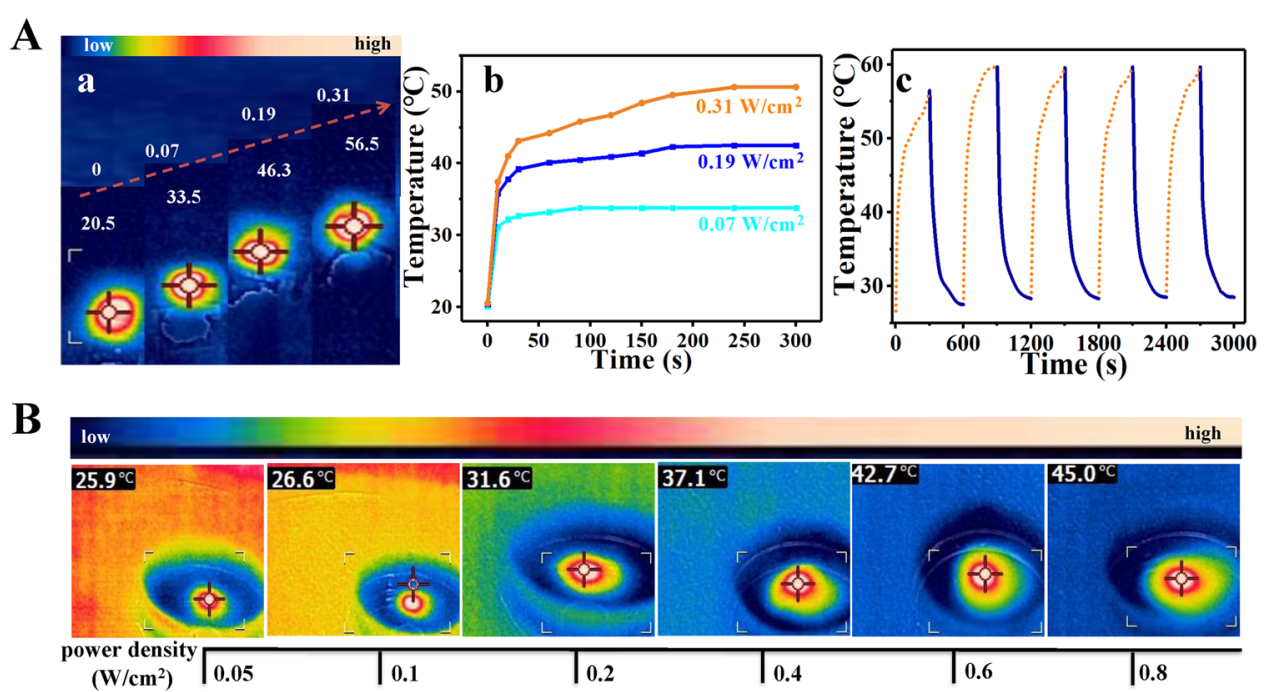

Fig. 4 Photothermal analysis. a (a) Thermal images of PG-Pg upon 5 min NIR laser irradiation with varying power densities. (b) Temperature profiles of PG-Pg irradiated by NIR lasers with varying power densities. (c) Temperature profile of PG-Pg irradiated by a 0.31 W/cm² laser applied at 5 min intervals. b IR images of PG-Pg hybrid hydrogel irradiated by laser with varying power densities $\left(0.05-0.8 \mathrm{~W} / \mathrm{cm}^{2}\right)$ in PBS.

densities required for samples to achieve 37.1, 42.7, and $45.0^{\circ} \mathrm{C}$ were $0.4,0.6$ and $0.8 \mathrm{~W} / \mathrm{cm}^{2}$, respectively. It is worth noting that the photothermal effect can be used to modulate the drug release profile ${ }^{34}$. Existing drug release and photothermal therapies for RA treatment mostly rely on the local injection of photothermal agents (e.g., nanoparticles $)^{35,36}$, and challenges remain in the local retention of nanoparticles and their dose-related toxicity. Our hybrid PG-Pg scaffold may hence provide an integrated solution for tissue replacement/repair and localized therapy.

\section{Drug-release functions of PG-Pg}

Three-dimensional water-swollen hydrogels have been extensively studied as drug delivery systems $\mathrm{s}^{37,38}$. Since the model drug (FL) was only embedded in the hydrogel component of the PG-Pg hybrid hydrogel system, we first studied the release profile from Pg hydrogels to better understand the release kinetics (see Fig. 5a, (a)). For all Pg hydrogels, the drug showed a burst release during the initial $4 \mathrm{~h}$, which gradually stabilized after $24 \mathrm{~h}$. The cumulative release increased with increasing glycerol content in the hydrogel. This result occurred because hydrogels containing a higher glycerol content undergo greater volume expansion, which allows faster molecular exchange. The drug release profile of Pg-5 h hydrogels is displayed in Fig. 5a, (b). Compared to Pg-0 and Pg-10, Pg0-5 h and Pg-10-5 h took longer to reach equilibrium. This may be because Pg-0-5 h and Pg-10-5 h underwent more significant swelling from their collapsed state, which disturbed the drug transport kinetics. The drug release rate increased with increasing irradiation laser power density (which is associated with increasing sample temperature). Figure $5 \mathrm{~b}$ compares the drug release profile of PG-Pg with that of Pg-20-5 h. The release rate slowed with the introduction of the reinforcing PCL-G-0.8 scaffold. This result may be due to the encapsulated scaffold serving as a physical barrier hindering the drug transport path. In Fig. 5c, the effect of NIR irradiation on drug release from the PG-Pg hybrid hydrogel was preliminarily explored at different time points $(10 \mathrm{~min}$ and $72 \mathrm{~h}$ after immersion). It can be seen that for both cases, higher temperatures (i.e., $42.7^{\circ} \mathrm{C}$ and $45.0^{\circ} \mathrm{C}$ ) due to laser irradiation were effective in upregulating drug release.

The potential drug elution mechanism from the hybrid hydrogel is presented in Fig. 5d. Under NIR irradiation, the laser-induced temperature rise in PG-Pg can lead to greater molecular mobility, hence accelerating the transport of both water and drug molecules and promoting drug release. The cumulative release may be enhanced and controlled by manipulating the laser power, which points to the possibility of achieving sustained and adjustable drug release through photothermal conversion.

\section{Protein adsorption and cell adhesion study}

To preserve the smoothness of the artificial cartilage surface and maintain its low friction in vivo, protein adsorption and cell adhesion on the hydrogel surface should be minimized. The protein adsorption on Pg-0 and Pg-20-5 h (immersed in newborn bovine serum overnight at $37^{\circ} \mathrm{C}$ ) was investigated by the detection of $\mathrm{N}$ on the sample surface using qualitative XPS analysis (shown in Fig. 6a). The literature suggests that proteins can only weakly adsorb onto a pristine PVA hydrogel (i.e. Pg-0) surface $^{39}$. The lower quantity of adsorbed proteins found 

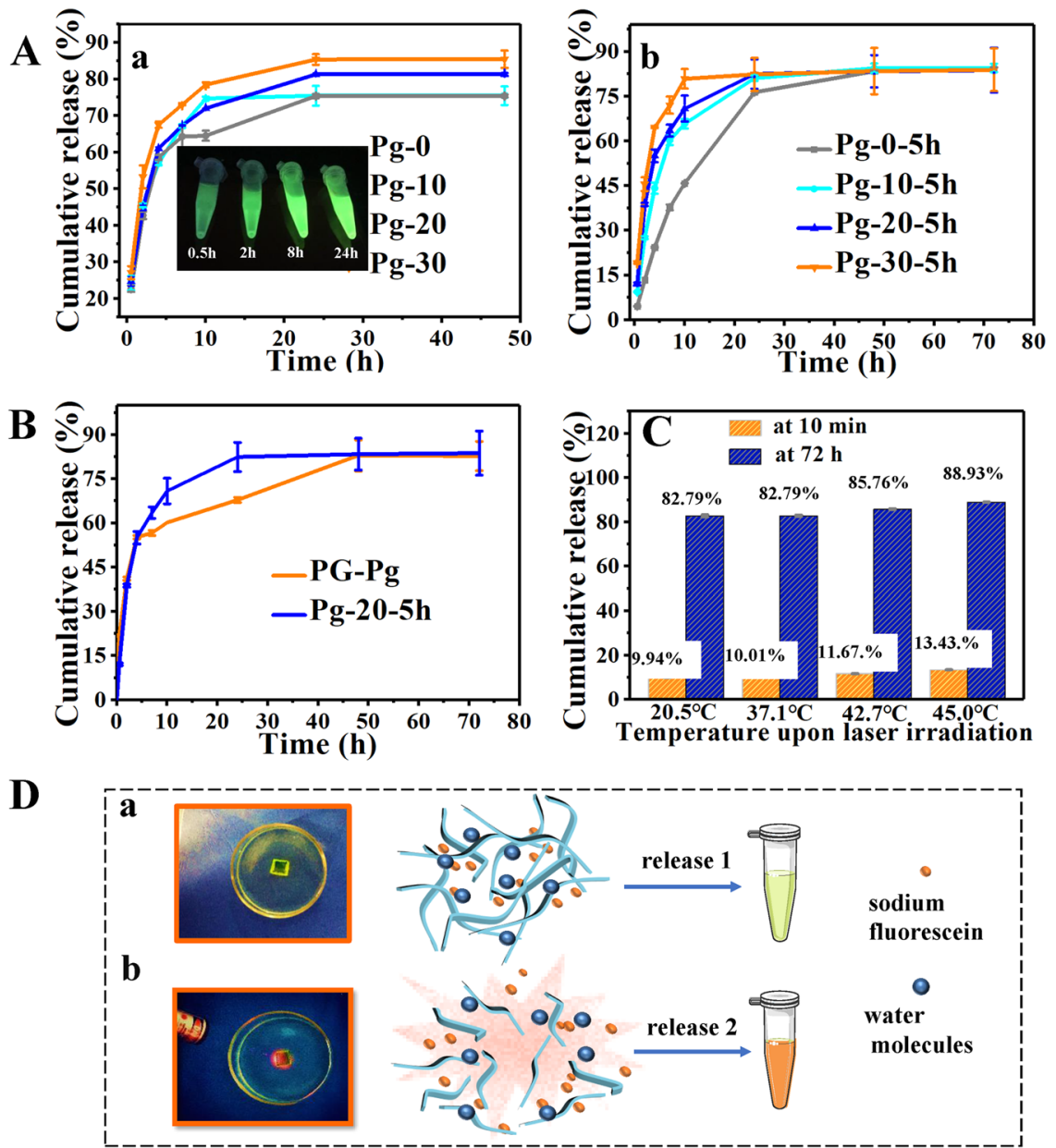

Fig. 5 Drug release study. a Drug release profile from (a) Pg hydrogels with varying glycerol contents and (b) Pg-5 h hydrogels with varying glycerol contents. b Drug release profile from Pg-20-5 h and PG-Pg. c Cumulative release from PG-Pg under laser irradiation at $10 \mathrm{~min}$ and $72 \mathrm{~h}$ after immersion in PBS. $\mathbf{d}$ Schematic showing the drug release mechanism of PG-Pg without NIR irradiation (a) and with NIR irradiation (b).

on Pg-20-5 h (Fig. 6a, (b) indicates that the Pg hydrogel has better resistance towards protein adsorption compared to pristine PVA, which favors its application as load-bearing cartilage.

In the subsequent cell experiment, chondrocyte cells were cultured on Pg-20-5 h for $48 \mathrm{~h}$, and cell adhesion was observed under SEM (Fig. 6b, (a)). Very few cells were found in the entire field, and all cells exhibited typical rounded chondrocyte morphology. This result was obtained because the highly lubricated Pg hydrogel surface resists cell adhesion ${ }^{40}$. Similar low cell adhesion was also observed for the PG-Pg hybrid hydrogel (see Fig. S5B). This result is as expected since the Pg hydrogel is fully encapsulated in the 3D printed PG scaffold (see Fig. S5A). CCK-8 testing was carried out to compare the proliferation of chondrocytes on the surface of Pg-0-5 h and Pg-30-5 h (see Fig. 6b, (b)). The absorbance values of all Pg hydrogel groups were slightly higher than those of the blank group. After 2 days of cultivation, the absorbance values of the Pg hydrogel groups decreased with increasing glycerol content, and the values of all hydrogel groups were much lower than those of the chondrocyte control group. The data for 5 days did not show significantly enhanced proliferation. A lack of cell induction is highly desirable for cartilage replacement materials to maintain their surface smoothness and hence preserve their long-term lubricity. Figure $6 \mathrm{c}$ shows the results of chondrocyte proliferation after culturing for 1 to 7 days in cell culture medium mixed with leaching solution. The results confirm that the noncytotoxicity of the PG-Pg hybrid hydrogel is comparable with that of the control group.

\section{Conclusions}

In summary, we have designed and fabricated a novel PG-Pg hybrid hydrogel with integrated photothermal conversion and drug release functions. By reinforcing glycerol-modified PVA with a 3D printed PCL-graphene 

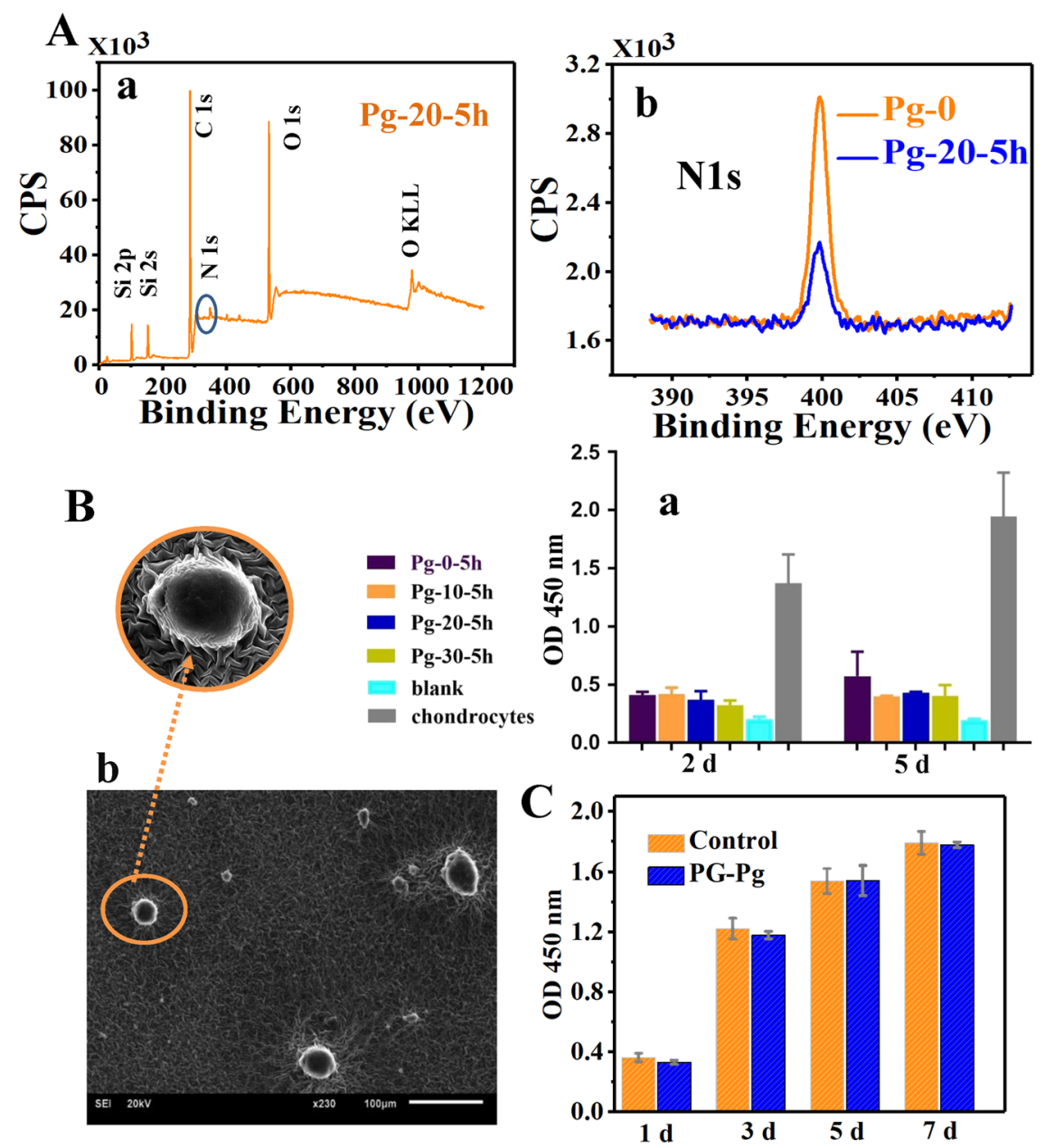

Fig. 6 Protein adsorption and cell adhesion study. a (a) XPS spectra of Pg-20-5 h. (b) N1s spectra of Pg-0 and Pg-20-5 h. b (a) SEM image of chondrocytes cultured for $48 \mathrm{~h}$ on Pg-20-5 h. (b) CCK-8 test of chondrocytes on the surfaces of hydrogels. The blank group refers to hydrogel and medium only (without cells). The chondrocyte group refers to a cell-only suspension without hydrogel. $\mathbf{c} 1 \mathrm{~d}$ to $7 \mathrm{~d}$ proliferation of chondrocytes cultured on PG-Pg.

composite scaffold with optimized composition and architecture, we achieved a hybrid hydrogel with desirable physical and mechanical properties (e.g., low friction, high water retention, and excellent stiffness, and toughness) comparable to those of natural load-bearing cartilage. The graphene-containing 3D printed reinforcing scaffold further provides a hybrid system with effective photothermal conversion properties, which can regulate drug release under external laser irradiation. The hybrid hydrogel system also allows for minimal protein adsorption and cell adhesion, further demonstrating its excellent potential for load-bearing cartilage applications.

\section{Acknowledgements}

The authors acknowledge funding support from the National Natural Science Foundation of China (No. 51673131, 51872190) and National Key Research and Development Program of China (2016YFA0201703/2016YFA0201700). The authors would also like to thank Suilin Liu, Hui Wang, Yi He, Shaolan Wang,
Aiqun Gu and Jiqiu Wen (Analytical \& Testing Center, SCU) for their help with XPS, SEM, DSC, and XRD testing.

\section{Author details}

${ }^{1}$ Analytical and Testing Center, State Key Laboratory of Oral Diseases, \& School of Materials Science and Engineering, Sichuan University, Chengdu 610065, China. ${ }^{2}$ School of Materials Science and Engineering, Southwest University of Science and Technology, Mianyang 621010, China. ${ }^{3}$ Advanced Composite Research Group (ACRG), School of Mechanical and Aerospace Engineering, Queen's University Belfast, Belfast BT9 5AH, UK

Conflict of interest

The authors declare that they have no conflict of interest.

Publisher's note

Springer Nature remains neutral with regard to jurisdictional claims in published maps and institutional affiliations.

Supplementary information is available for this paper at https://doi.org/ 10.1038/s41427-020-0199-6. 
Received: 5 September 2019 Revised: 23 December 2019 Accepted: 25 December 2019.

Published online: 21 February 2020

\section{References}

1. Kujala, U. M. et al. Knee osteoarthritis in former runners, soccer players, weight lifters, and shooters. Arthritis Rheum. 38, 539-546 (1995).

2. Zion Market Research. Global cartilage repair regeneration market will reach USD 6.5 billion by 2024: zion market research. https://www.orthoconnections. $\mathrm{com} /$ news/global-cartilage-repair-regeneration-market-will-reach-usd-6-5billion-by-2024-zion-market-research (2018).

3. D'Este, M., Eglin, D. \& Alini, M. Lessons to be learned and future directions for intervertebral disc biomaterials. Acta Biomater. 78, 13-22 (2018).

4. Liu, M. et al. Injectable hydrogels for cartilage and bone tissue engineering Bone Res. 5, 17014 (2017).

5. Koo, Y. et al. 3D printed cell-laden collagen and hybrid scaffolds for in vivo articular cartilage tissue regeneration. J. Ind. Eng. Chem. 66, 343-355 (2018).

6. Chuang, E.-Y., Chiang, C.-W., Wong, P.-C. \& Chen, C.-H. Hydrogels for the application of articular cartilage tissue engineering: a review of hydrogels. Adv. Mater. Sci. Eng. 2018, 1-13 (2018).

7. Wang, Z. et al. 3D-printable self-healing and mechanically reinforced hydrogels with host-guest non-covalent interactions integrated into covalently linked networks. Mater. Horiz. 6, 733-742 (2019).

8. Jeffery, A. K., Blunn, G. W., Archer, C. W. \& Bentley, G. Three-dimensional collagen architecture in bovine articular cartilage. J. Bone Jt. Surg. Br. 73-B, 795-801 (1991).

9. Abbott, A. Biology's new dimension. Nature 424, 870 (2003).

10. Visser, J. et al. Reinforcement of hydrogels using three-dimensionally printed microfibres. Nat. Commun. 6, 6933 (2015).

11. Lin, S. et al. Design of stiff, tough and stretchy hydrogel composites via nanoscale hybrid crosslinking and macroscale fiber reinforcement. Soft Matter 10, 7519-7527 (2014).

12. Yodmuang, S. et al. Silk microfiber-reinforced silk hydrogel composites for functional cartilage tissue repair. Acta Biomater. 11, 27-36 (2015).

13. Benjamin, M. \& Evans, E. J. Fibrocartilage. J. Anat. 171, 1-15 (1990).

14. Heo, D. N. et al. Enhanced bone tissue regeneration using a 3D printed microstructure incorporated with a hybrid nano hydrogel. Nanoscale $\mathbf{9}$, 5055-5062 (2017).

15. Ridzwan, M., Shuib, S., Hassan, A., Shokri, A. \& Ibrahim, M. M. Problem of stress shielding and improvement to the hip implant designs: a review. J. Dent. Res. 7, 460-467 (2007)

16. Chen, J., Shi, X., Ren, L. \& Wang, Y. Graphene oxide/PVA inorganic/organic interpenetrating hydrogels with excellent mechanical properties and biocompatibility. Carbon 111, 18-27 (2017).

17. Jing, L. et al. Biocompatible hydroxylated boron nitride nanosheets/poly(vinyl alcohol) interpenetrating hydrogels with enhanced mechanical and thermal responses. ACS Nano 11, 3742-3751 (2017).

18. Bodugoz-Senturk, H., Macias, C. E., Kung, J. H. \& Muratoglu, O. K. Poly(vinyl alcohol)-acrylamide hydrogels as load-bearing cartilage substitute. Biomaterials 30, 589-596 (2009).

19. Li, J., Suo, Z. \& Vlassak, J. J. Stiff, strong, and tough hydrogels with good chemical stability. J. Mater. Chem. B 2, 6708-6713 (2014).

20. Shi, Y. \& Xiong, D. Microstructure and friction properties of PVA/PVP hydrogels for articular cartilage repair as function of polymerization degree and polymer concentration. Wear 305, 280-285 (2013).
21. Wang, W., Huang, B., Byun, J. J. \& Bártolo, P. Assessment of PCL/carbon material scaffolds for bone regeneration. J. Mech. Behav. Biomed. Mater. 93, 52-60 (2019).

22. Li, Y., Wang, S., Wang, Q. \& Xing, M. A comparison study on mechanical properties of polymer composites reinforced by carbon nanotubes and graphene sheet. Compos. Part B-Eng. 133, 35-41 (2018).

23. Feng, L., Wu, L. \& Qu, X. New horizons for diagnostics and therapeutic applications of graphene and graphene oxide. Adv. Mater. 25, 168-186 (2013).

24. Zheng, X., Fu, Z., Du, K., Wang, C. \& Yi, Y. Minimal surface designs for porous materials: from microstructures to mechanical properties. J. Mater. Sci. $\mathbf{5 3}$ 10194-10208 (2018)

25. Liu, T. et al. Hydrogen-bonded polymer-small molecule complexes with tunable mechanical properties. Macromol. Rapid Commun. 39, e1800050 (2018).

26. Kim, D. S. et al. Preparation of ion exchange membranes for fuel cell based on crosslinked poly(vinyl alcohol) with poly(styrene sulfonic acid-co-maleic acid). J. Membr. Sci. 281, 156-162 (2006).

27. Assender, H. E. \& Windle, A. H. Crystallinity in poly(vinyl alcohol). 1. An X-ray diffraction study of atactic PVOH. Polymer 39, 4295-4302 (1998).

28. Ricciardi, R., Auriemma, F., De Rosa, C. \& Lauprêtre, F. X-ray diffraction analysis of poly(vinyl alcohol) hydrogels, obtained by freezing and thawing techniques. Macromolecules 37, 1921-1927 (2004).

29. Peng, M., Xiao, G., Tang, X. \& Zhou, Y. Hydrogen-bonding assembly of rigid-rod poly(p-sulfophenylene terephthalamide) and flexible-chain poly(vinyl alcohol) for transparent, strong, and tough molecular composites. Macromolecules 47, 8411-8419 (2014)

30. Gwon, H.J. et al. Characterization of PVAvglycerin hydrogels made by $\gamma^{-}$ irradiation for advanced wound dressings. Korean J. Chem. Eng. 26, 1686-1688 (2010).

31. Chang, J. Y. et al. Biopolymers PVA Hydrogels Anionic Polymerisation Nanocomposites. Vol. 153 (Springer Science \& Business Media, 2000)

32. Baker, M. I., Walsh, S. P., Schwartz, Z. \& Boyan, B. D. A review of polyvinyl alcohol and its uses in cartilage and orthopedic applications. J. Biomed. Mater. Res. B 100, 1451-1457 (2012).

33. Zhang, S. et al. Effect of magnetic nanoparticles size on rheumatoid arthritis targeting and photothermal therapy. Colloids Surf, B: Biointerfaces 170, 224-232 (2018).

34. Gu, Z., Zhu, S., Yan, L., Zhao, F. \& Zhao, Y. Graphene-based smart platforms for combined cancer therapy. Adv. Mater. 31, e1800662 (2019).

35. Lee, S.M. et al. Targeted chemo-photothermal treatments of rheumatoid arthritis using gold half-shell multifunctional nanoparticles. ACS Nano 7, 50-57 (2013).

36. Pirmardvand Chegini, S., Varshosaz, J. \& Taymouri, S. Recent approaches for targeted drug delivery in rheumatoid arthritis diagnosis and treatment. Artif. Cells Nanomed. Biotechnol. 46, 502-514 (2018).

37. Talebian, S. et al. Biopolymers for antitumor implantable drug delivery systems: recent advances and future outlook. Adv. Mater. 30, e1706665 (2018).

38. Joshi, N. et al. Towards an arthritis flare-responsive drug delivery system. Nat. Commun. 9, 1275 (2018)

39. Liu, Y., Vrana, N. E., Cahill, P. A. \& McGuinness, G. B. Physically crosslinked composite hydrogels of PVA with natural macromolecules: structure, mechanical properties, and endothelial cell compatibility. J. Biomed. Mater. Res B 90, 492-502 (2009).

40. Zhang, C. et al. High-performance photopolymerized poly(vinyl alcohol)/silica nanocomposite hydrogels with enhanced cell adhesion. ACS Appl. Mater. Interfaces 10, 27692-27700 (2018). 